\title{
SOME BASIC THEOREMS IN DIFFERENTIAL ALGEBRA (CHARACTERISTIC $p$, ARBITRARY)
}

\author{
BY \\ A. SEIDENBERG
}

J. F. Ritt [6] has established for differential algebra of characteristic 0 a number of theorems very familiar in the abstract theory, among which are the theorems of the primitive element, the chain theorem, and the Hilbert Nullstellensatz. Below we also consider these theorems for characteristic $p \neq 0$, and while the case of characteristic 0 must be a guide, the definitions cannot be taken over verbatim. This usually requires that the two cases be discussed separately, and this has been done below. The subject is treated $a b$ initio, and one may consider that the proofs in the case of characteristic 0 are being offered for their simplicity. In the field theory, questions of separability are also considered, and the theorem of S. MacLane on separating transcendency bases [4] is established in the differential situation.

1. Definitions. By a differentiation over a ring $R$ is meant a mapping $u \rightarrow u^{\prime}$ from $R$ into itself satisfying the rules $(u v)^{\prime}=u v^{\prime}+u^{\prime} v$ and $(u+v)^{\prime}$ $=u^{\prime}+v^{\prime}$. A differential ring is the composite notion of a ring $R$ and a differentiation over $R$ : if the ring $R$ becomes converted into a differential ring by means of a differentiation $D$, the differential ring will also be designated simply by $R$, since it will always be clear which differentiation is intended. If $R$ is a differential ring and $R$ is an integral domain or field, we speak of a differential integral domain or differential field respectively. An ideal $A$ in a differential ring $R$ is called a differential ideal if $u \in A$ implies $u^{\prime} \in A$. The ring $\{u+A\}$ of residue classes of the differential ring $R \bmod$ a differential ideal $A$ is also a differential ring under the differentiation $(u+A)^{\prime}=u^{\prime}+A$.

If $F$ is a differential field, then from $(v \cdot u / v)^{\prime}=v(u / v)^{\prime}+v^{\prime}(u / v)$ we obtain $(u / v)^{\prime}=\left(u^{\prime} v-u v^{\prime}\right) / v^{2}$. If $R$ is a differential integral domain, then its quotient field $F$ becomes a differential field on setting $(u / v)^{\prime}=\left(u^{\prime} v-u v^{\prime}\right) / v^{2}$ : it is this differential field which is intended when we speak of the quotient field of $R$. From $1^{\prime}=(1 \cdot 1)^{\prime}=1 \cdot 1^{\prime}+1^{\prime} \cdot 1$, it follows that $1^{\prime}=0$, whence the differentiation in $F$ coincides with the given differentiation in $R$.

An element $u$ whose derivative $u^{\prime}$ is zero will be called a constant. It is immediate that all the elements of the primitive field of a differential field are constants.

The modifier "differential" will usually be omitted: if "differential" is not intended, we shall refer to the modified object "in the algebraic sense."

Notation. The symbols ( ) and [ ] will be used in their usual senses of field and ring adjunction respectively: the symbols \langle\rangle and \{\} will be

Presented to the Society, May 3, 1952; received by the editors December 14, 1951. 
used in the corresponding differential situations. The symbol ( ) is also used in its usual ideal-theoretic sense, the symbol [ ] taking its place in the differential case: thus, for example, $[p, q]=\left(p, q, p^{\prime}, q^{\prime}, p^{\prime \prime}, q^{\prime \prime}, \cdots\right)$. The symbol $\{\ldots\}$ is also used in the set-theoretic sense, to indicate the set exhibited, and will also be used to indicate the perfect ideal (definition below) generated by the elements enclosed. Subscripts are used in the usual way, but are also used to indicate derivatives: thus $u, u_{1}, u_{2}, \cdots$ may indicate the successive derivatives of $u$; but also $u_{1}, u_{2}, \cdots, u_{n}$ may indicate several quantities not particularly related, in which case a second subscript is used to indicate the derivative.

2. Field extensions. Let $K$ be a differential field and $F$ a subfield of $K$; i.e., $F$ is understood to be a differential field under a differentiation induced in it by the given differentiation in $K$. Let $F\{u\}$ designate the smallest differential ring containing $F$ and an element $u \in K ; F\langle u\rangle$ designates the smallest differential field containing $F$ and $u$.

Let now $K$ be of characteristic 0 . We shall say that the element $u \in K$ is algebraic over $F$ if there is a nontrivial polynomial relation $H\left(u, u^{\prime}, u^{\prime \prime}, \cdots, u^{(i)}\right)$ $=0$ satisfied by $u$ and its first $i$ derivatives (for some $i$ ); that is, if $U, U_{1}, U_{2}, \cdots$ is a sequence of indeterminates (in the usual algebraic sense),

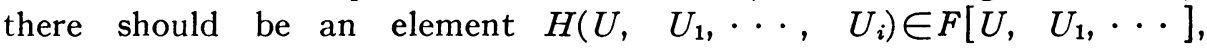
$H\left(U, U_{1}, \cdots, U_{i}\right) \neq 0$, such that $H\left(u, u^{\prime}, u^{\prime \prime}, \cdots, u^{(i)}\right)=0$.

In van der Waerden's Moderne Algebra [7], the theory of linear as well as algebraic dependence is made to rest on the following axioms:

I. $u_{i}$ is dependent on $u_{1}, \cdots, u_{n}$.

II. If $u$ is dependent on $u_{1}, \cdots, u_{n}$, but not on $u_{1}, \cdots, u_{n-1}$, then $u_{n}$ is dependent on $u_{1}, \cdots, u_{n-1}, u$.

III. If $w$ is dependent on $v_{1}, \cdots, v_{m}$ and each $v_{j}$ is dependent on $u_{1}, \cdots, u_{n}$, then $w$ is dependent on $u_{1}, \ldots, u_{n}$.

If, now, $y$ dependent on $x_{1}, \cdots, x_{n}$ is taken to mean that $y$ is algebraic over $F\left\langle x_{1}, \cdots, x_{n}\right\rangle$, then the above axioms become true theorems. The first two are trivial, and the third follows at once from the following lemma.

Lemma. If $u$ is algebraic over $F$, then $F\langle u\rangle=F\left(u, u^{\prime}, u^{\prime \prime}, \cdots\right)$ has a finite degree of transcendency over $F$; in fact $F\left(u, u^{\prime}, \ldots\right)$ is even finite over $F$. On the other hand, if $u$ is not algebraic over $F$, then $F\langle u\rangle$ has infinite degree of transcendency over $F$.

Proof. Let $u$ be algebraic over $F$; then there is an $r>0$ such that $u=u^{(0)}$, $u^{(1)}, u^{(2)}, \cdots, u^{(r)}$ are algebraically dependent (in the algebraic sense) over $F$, while $u, u^{(1)}, \cdots, u^{(r-1)}$ are not. Let $G\left(U, U^{(1)}, \cdots, U^{(r)}\right)$ be a polynomial of least degree in $U^{(r)}$ which is satisfied by $u, u^{(1)}, \cdots, u^{(r)}$ over $F$. Let $S\left(U, \cdots, U^{(r)}\right)=\partial G / \partial U^{(r)}$ : we see that $S\left(u, \cdots, u^{(r)}\right) \neq 0$. Now $S$ is the coefficient of $U^{(r+1)}$ in $\left(G\left(U, U^{(1)}, \cdots, U^{(r)}\right)\right)^{\prime}$, whence we see that $u^{(r+1)} \in F\left(u, u^{(1)}, \cdots, u^{(r)}\right)$, and likewise $u^{(j)} \in F\left(u, \cdots, u^{(r)}\right)$ for $j>r+1$ : 
this proves the first point. The second point is trivial.

Statement III is now immediate; for $F\langle u, v, w\rangle$ has a finite degree of transcendency over $F\langle u, v\rangle, F\langle u, v\rangle$ has a finite degree of transcendency over $F\langle u\rangle$, whence $F\langle u, v, w\rangle$ and $F\langle u, w\rangle$ have finite degrees of transcendency over $F\langle u\rangle$.

The whole theory of degree of transcendency in the algebraic case can therefore be carried over to the differential case, and we can speak of the degree of differential transcendency (d.d.t.). In particular note that if $u$ and $v$ are algebraic over $F$, then so are $u \pm v, u \cdot v$, and $u / v$ (if $v \neq 0$ ).

The above results are, of course, well known, and go back to $\mathrm{H}$. W. Raudenbush [5].

For any $n$ it is clear that we can construct a differential ring $F\left\{U_{1}, \cdots, U_{n}\right\}$ with d.d.t $F\langle U\rangle / F=n$ : then $U_{1}, \cdots, U_{n}$ will be called differential indeterminates, as in the algebraic case.

3 . The theorem of the primitive element. This theorem is entirely parallel to the algebraic theorem. It asserts that if $u, v$ are algebraic over $F$, then under certain conditions $F\langle u, v\rangle=F\langle\theta\rangle$. The proofs in the algebraic case depend on the fact that if $G\left(X_{1}, \cdots, X_{n}\right)$ is a polynomial different from 0 and $F$ is an infinite field, then there exist $x_{i} \in F, i=1, \cdots, n$, such that $G\left(x_{1}, \cdots, x_{n}\right) \neq 0$. If we consider the differential polynomial $X^{\prime}$, then it becomes clear that a like fact could obtain over a differential field $F$ only if $F$ contains nonconstant elements. Conversely, if $F$ does contain nonconstants, and $G\left(X_{1}, \cdots, X_{n}\right) \neq 0$ is a differential polynomial, then there exist $x_{i} \in F, i=1, \cdots, n$, such that $G\left(x_{1}, \cdots, x_{n}\right) \neq 0$ : we refer to Ritt $[6$, p. 35] for the proof. Moreover, the theorem of the primitive element could not hold, in general, if all elements of $F$ were constants: for let $u, v$ be two indeterminates in the algebraic sense and convert $F(u, v)$ into a differential field by setting every derivative equal to 0 . Then $F\langle u, v\rangle=F(u, v), F\langle\theta\rangle=F(\theta)$, and $F(u, v)=F(\theta)$ is clearly impossible. Subject to these necessary conditions, the theorem holds.

THEOREM 1. Let $F$ contain nonconstant elements. If $u$, $v$ are algebraic over $F$, then there exists $a \lambda \in F$ such that $F\langle u, v\rangle=F\langle u+\lambda v\rangle$. (Note that $F$ is for the present of characteristic 0 .)

Proof. Construct $F\langle u, v\rangle\langle\Lambda\rangle$, where $\Lambda$ is a differential indeterminate. Since $u, v, \Lambda$ are algebraic over $F\langle\Lambda\rangle$, we have that $u+\Lambda v$ is algebraic over $F\langle\Lambda\rangle$, and hence we have a nontrivial polynomial relation:

$$
G\left(\Lambda, \Lambda^{(1)}, \cdots, \Lambda^{(t)},(u+\Lambda v),(u+\Lambda v)^{(1)}, \cdots,(u+\Lambda v)^{(s)}\right)=0 .
$$

Here we are supposing that $s$ is as small as possible and that $G$ is of least possible degree in $(u+\Lambda v)^{(s)}$. Let $u+\Lambda v=w$ : note that $\partial w^{(i)} / \partial \Lambda^{(s)}=0$ if $i<s$, and $=v$ if $i=s$. Taking the partial of the above relation with respect to $\Lambda^{(s)}$ we obtain: 


$$
\frac{\partial G}{\partial \Lambda^{(s)}}+\frac{\partial G}{\partial w^{(s)}} \cdot v=0 .
$$

Because of the minimal conditions placed on $G$, we have $\partial G / \partial w^{(s)}=S(\Lambda, u+\Lambda v)$ $\neq 0$, whence $v \in F\langle\Lambda\rangle\langle u+\Lambda v\rangle$ : we now specialize, appropriately, $\Lambda \rightarrow \lambda \in F$; some care has to be exercised, as we cannot suppose (as in the algebraic theorem) that $v$ can be written with a denominator free of $w$. By the lemma of Ritt mentioned we could specialize $\Lambda$ to $\lambda \in F\langle u, v\rangle$ so that $S(\lambda, u+\lambda v) \neq 0$ : actually, the proof of the lemma shows that if $\xi$ is a nonconstant, then one can specialize $\Lambda$ to a polynomial in $\xi$ with rational numbers as coefficients (the main point of this proof will have to be considered explicitly below in discussing the case of characteristic $p \neq 0)$. Thus we may take $\lambda \in F$, whence $v \in F\langle u+\lambda v\rangle$ and $F\langle u+\lambda v\rangle=F\langle u, v\rangle$. (Or one can apply the lemma directly by writing $1 / S(\Lambda, u+\Lambda v)$ in the form $C(\Lambda, u, v) / D(\Lambda, u, v)$, where $C, D$ are polynomials and in $D$ occur only $u_{i}$ and $v_{j}$ in some selected transcendency basis of $F\langle u, v\rangle / F$ : one would then have only to specialize $\Lambda$ so that some one coefficient of $D$ does not vanish.)

For a previous treatment of the above theorem, see E. R. Kolchin [2].

4. The Hilbert Nullstellensatz. Let $R=F\left\{U_{1}, \cdots, U_{n}\right\}$ be a polynomial ring in $n$ differential indeterminates. By a point one means a system $u_{1}, \cdots, u_{n}$ of differential quantities in an extension field of $F$; by a zero of an ideal $A$ one means a point annihilating all the elements of $A$. By the variety $V(A)$ of an ideal $A$ one means the set of zeros of $A$. The Hilbert Nullstellensatz says that if $G \in R$ vanishes over $V(A)$, then $G^{\rho} \in A$ for some $\rho$. Another possible definition of $V(A)$ restricts $V(A)$ to consist of the 0 -dimensional points, i.e., points $\left(u_{1}, \cdots, u_{n}\right)$ such that d.d.t. $F\left\langle u_{1}, \cdots, u_{n}\right\rangle / F$ $=0$. According as one takes the first or second definition one speaks of the weak or strong form of the theorem: initially we are concerned only with the weak form. Another remark must be made: exception can be taken to the above definition of $V(A)$ since no bound has been placed on the cardinal number of $V(A)$. This quite valid objection is easily overcome as it is sufficient to have a field $K \supseteq F$ which contains for every prime $P$ in $R$ the coördinates of a general point of $P$, i.e., a point $u_{1}, \cdots, u_{n}, u_{i} \in K$, such that $R / P \cong F\left\{u_{1}, \cdots, u_{n}\right\}$, and one will then define point to have coördinates in $K$. It would be easy to construct $K$, but we need not be detained over this matter, since Hilbert's Theorem has an obviously equivalent form stated directly in terms of $R$, namely, that the set $\left\{G \mid G^{\rho} \in A\right\}=\cap P$ over the prime ideals $P$ in $R$ which contain $A$.

By a perfect ideal $A$ one means an ideal such that $G^{\circ} \in A$ implies $G \in A$. One consequence of Hilbert's Theorem is that if $A$ is an ideal, then the set $\left\{G \mid G^{\rho} \in A\right\}$ is an ideal. Hence it is clear that Hilbert's Theorem cannot hold without some modification if $F$ is of characteristic $p \neq 0$, since, e.g., for $n=1$, we have $u^{\prime \rho} \notin\left[u^{p}\right]=A$, i.e., $\left\{G \mid G^{\rho} \in\left[u^{p}\right]\right\}$ is not an ideal. This possibility 
does not arise in the case of ordinary polynomial rings, and in fact it is well known that $\left\{G \mid G^{\rho} \in A\right\}$ is an ideal if $A$ is; neither does it arise in the differential case if the characteristic is 0 , since one shows by a simple calculation, which depends upon the characteristic, however, that $\left(G^{\prime}\right)^{2 \rho-1} \in\left[G^{\rho}\right]$. We may thus say that Hilbert's Theorem consists of two parts, the first says that $\left\{G \mid G^{\rho} \in A\right\}$ is an ideal, in which case it is obviously perfect, and the second says that a perfect ideal is the intersection of prime ideals. This second part is true in general, and deserves the name of Hilbert.

Hilbert's Nullstellensatz (weak form). A perfect ideal in the polynomial ring $F\left\{U_{1}, \cdots, U_{n}\right\}$ is the intersection of prime ideals ( $F$ of arbitrary characteristic).

Proof. Let $A$ be the given perfect ideal, and let $\alpha \in R, \alpha \notin A$. We have to show the existence of a prime ideal $P$ containing $A$ but not containing $\alpha$. Let then $S$ be the set of perfect ideals containing $A$ but not meeting the multiplicatively closed system $\left\{\alpha^{\rho} \mid \rho=1,2, \cdots\right\}$. Partially ordering $S$ by inclusion, we see by Zorn's Lemma that there is maximal element $P$ in $S$, and this we claim is prime. In fact, let $u, v \in R, u \notin P, v \notin P$, but $u v \in P$. Let $\{P, u\},\{P, v\}$ be the smallest perfect ideals containing $[P, u],[P, v]$ respectively. Some power of $\alpha$ lies in $\{P, u\}$, and some power in $\{P, v\}$, by the maximality of $P$. In the case of characteristic 0 , by the remark that $\{B\}=\left\{G \mid G^{\rho} \in[B]\right\}$, one would even have that a power of $\alpha$ is in $[P, u]$, and a power in $[P, v]$. Now $u v \in P$ implies $\left(u^{\prime} v+u v^{\prime}\right) u^{\prime} v \in P$, whence $\left(u^{\prime} v\right)^{2} \in P$, $u^{\prime} v \in P$, and more generally $u^{(i)} v^{(j)} \in P$. Hence $[P, u] \cdot[P, v] \subseteq P$, so a power of $\alpha$ is in $P$, contradiction. In the case of arbitrary characteristic it remains to prove the following lemma. Kolchin $[3, \S 3]$ has this result, but it is well, for later purposes, to have a proof before our eyes.

Lemma. If $u v$ is in the perfect ideal $P$, then $\{P, u\} \cdot\{P, v\} \subseteq P$.

Proof. Let $A_{0}=[P, u]$, let $A_{1}=$ the differential ideal generated by the elements $G$ such that $G^{\rho} \in A_{0}$ for some $\rho$, and let $A_{i}$ be defined recursively as the ideal generated by the $G$ such that $G^{\rho} \in A_{i-1}$ for some $\rho$; define $B_{0}=[P, v]$, $B_{1}, B_{2}, \cdots$ similarly. We have $A_{0} \subseteq\{P, u\}$, whence also $A_{1} \subseteq\{P, u\}$, and now inductively that $A_{i} \subseteq\{P, u\}$. Hence $\cup A_{i} \subseteq\{P, u\}$. Conversely, let $G^{\rho} \in \cup A_{i}$; then $G^{\rho} \in A_{i}$, for some $i$, so $G \in A_{i+1}$, whence $G \in \cup A_{i}$, i.e., $\cup A_{i}$ is a perfect ideal, so $\{P, u\} \subseteq \cup A_{i}$. Hence $\{P, u\}=\bigcup A_{i}$ and similarly $\{P, v\}$ $=\mathrm{U} B_{i}$. We have seen already that $A_{0} \cdot B_{0} \subseteq P$; the proof will be complete upon showing that $A_{i} \cdot B_{i} \subseteq P$. We use induction. The ideal $A_{i}$ is generated

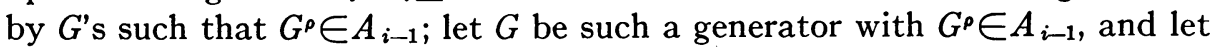
$H$ similarly be a generator of $B_{i}$, say $H^{\sigma} \in B_{i-1}$. Then $(G H)^{\rho \sigma} \in A_{i-1} B_{i-1} \subseteq P$, so $G H \in P$, and also $G^{(i)} H^{(j)}$ by a previous calculation. Hence $A_{i} \cdot B_{i} \subseteq P$, and the proof is complete.

The following theorem, taken in conjunction with the above, amounts to 
the strong Nullstellensatz. Since dimension has so far been considered only for characteristic 0 , the theorem is at this point necessarily subject to that restriction.

Hilbert's NUllstellensatz (STRONG FORM). Let $P$ be an $r$-dimensional prime ideal in $R=F\left\{U_{1}, \cdots, U_{n}\right\}, r>0$; i.e., d.d.t. $F\left\langle u_{1}, \cdots, u_{n}\right\rangle / F=r$, where $F\left\{u_{1}, \cdots, u_{n}\right\}=R / P$. Let $\alpha \in R, \alpha \notin P$. Then there exists an $(r-1)$ dimensional prime ideal $Q, P \subset Q$, such that $\alpha \notin Q$.

Below, in $\S 8$, we offer a very brief proof of this theorem. The following proof introduces two points not strictly necessary for a proof. One is the following lemma. In the case $n=1$, if $P$ is a proper prime ideal, then $R / P$ $=F\{u\}$, and $F\langle u\rangle=F\left(u, u_{1}, \cdots, u_{r}\right)$ is a field of algebraic functions of $r$ variables, $u, u_{1}, \cdots, u_{r-1}$ algebraically independent over $F$. The lemma is the converse.

LEMma. Let $F\left(u, u_{1}, \cdots, u_{r}\right)$ be a field of algebraic functions of $r$ variables, with $u_{r}$ algebraic and separable over $F\left(u, u_{1}, \cdots, u_{r-1}\right) ; F$, a differential field. Then there is one and only one way to convert $F\left(u, \cdots, u_{r}\right)$ into a differential field so that $u^{\prime}=u_{1}, u_{1}^{\prime}=u_{2}, \cdots, u_{r-1}^{\prime}=u_{r}$.

This is a well known result; see for example [8, chap. I, Prop. 15, p. 12].

Proof of the theorem. We may suppose that $u_{1}, \cdots, u_{r}$ are algebraically independent over $F$. Since $r>0, F\left\langle u_{1}, \cdots, u_{r}\right\rangle$ contains nonconstant elements, so there exists a $w$ such that $F\left\langle u_{1}, \cdots, u_{r}, w\right\rangle=F\left\langle u_{1}, \cdots, u_{n}\right\rangle$. The element $w$ satisfies an equation $G\left(U_{1}, \cdots, U_{r} ; W, W_{1}, \cdots, W_{t+1}\right)=0$, where $t$ is as small as possible and $G$ is irreducible in $F\left\{U_{1}, \cdots, U_{r}\right\}\left[W, \cdots, W_{t+1}\right]$. Note that if $H(U ; W)$ is in this last ring and $H(u ; w)=0$, then $H$ is divisible by $G$ in that ring. Let $S=\partial G / \partial W_{t+1}$. We have $u_{r+i}$ $=f_{i}\left(u_{1}, \cdots, u_{r} ; w\right) / d\left(u_{1}, \cdots, u_{r} ; w, \cdots, w_{t}\right)$, where $f_{i}, d \in F\left\{U_{1}, \cdots, U_{r} ; W\right\}$. Let $1 / S(u ; w)=g\left(u_{1}, \cdots, u_{r} ; w, \cdots, w_{t+1}\right) / e\left(u_{1}, \cdots, u_{r} ; w, \cdots, w_{t}\right)$ and let $1 / \alpha\left(u_{1}, \cdots, u_{n}\right)=h\left(u_{1}, \cdots, u_{r} ; w\right) / e_{1}\left(u_{1}, \cdots, u_{r} ; w, \cdots, w_{t}\right)$, where $g, h, e, e_{1} \in F\left\{U_{1}, \cdots, U_{r} ; W\right\}$, and, moreover, we may write $d=e=e_{1}$; note that $S g-e$ is divisible by $G$. We now define a differential field by binding $U_{1}, \cdots, U_{r}$, but in such manner that not all the coefficients of $d\left(U_{1}, \cdots, U_{r} ; W\right)$, regarded as a polynomial in $W, \cdots, W_{t}$, become equal to zero, i.e., we have a field $F\left\langle\bar{u}_{1}, \cdots, \bar{u}_{r}\right\rangle$, of d.d.t. $r-1$ over $F$, and such that $d\left(\bar{u}_{1}, \cdots, \bar{u}_{r} ; W\right) \neq 0$; moreover, we can also require, and do, that $G\left(\bar{u}_{1}, \cdots, \bar{u}_{r} ; W, \cdots, W_{t+1}\right)$ be of positive degree in $W_{t+1}$. Then $G\left(\bar{u}_{1}, \cdots, \bar{u}_{r} ; W, \cdots, W_{t+1}\right)$ will have an irreducible factor over $F\left\langle\bar{u}_{1}, \cdots, \bar{u}_{r}\right\rangle$ of positive degree in $W_{t+1}$, and through it we define, in the canonical manner, a differential field $F\left\langle\bar{u}_{1}, \cdots, \bar{u}_{r} ; \bar{w}\right\rangle$; it will then be so that $\bar{d}=d\left(\bar{u}_{1}, \cdots, \quad \bar{u}_{r} ; \quad \bar{w}\right) \neq 0$ and $\bar{S}=S\left(\bar{u}_{1}, \cdots, \quad \bar{u}_{r} ; \bar{w}\right) \neq 0$. Define $\bar{u}_{r+i}=f_{i}(\bar{u}, \bar{w}) / d(\bar{u}, \bar{w})$. We now prove that $H\left(u_{1}, \cdots, u_{n} ; w\right)=0$ implies $H\left(\bar{u}_{1}, \cdots, \bar{u}_{n} ; \bar{w}\right)=0$; in particular it will therefore be true that 
$\alpha\left(\bar{u}_{1}, \cdots, \bar{u}_{n}\right) \neq 0$. Let $(d(U, W))^{\rho} H\left(U_{1}, \cdots, U_{n} ; W\right) \rightarrow H_{1}\left(U_{1}, \cdots, U_{r} ; W\right)$ under the substitution $U_{r+i} \rightarrow f_{i}\left(U_{1}, \cdots, U_{r} ; W\right) / d$, where $\rho$ is chosen sufficiently high that $H_{1}$ be a polynomial. Further, let $(S(U, W))^{\sigma} H_{1}$ $\equiv H_{2}\left(U_{1}, \cdots, U_{r} ; W, \cdots, W_{t+1}\right) \bmod [G]$. It is sufficient to prove $H_{2}\left(\bar{u}_{1}, \cdots, \bar{u}_{r} ; \bar{w}\right)=0$, and we have $H_{2}\left(u_{1}, \cdots, u_{r} ; w\right)=0$. But $H_{2}(u ; w)=0$ implies $H_{2}(U, W)=A(U, W) \cdot G(U, W)$, whence $H_{2}(\bar{u}, \bar{w})=A(\bar{u}, \bar{w}) \cdot G(\bar{u}, \bar{w})$ $=0$. If $\bar{u}_{1}, \cdots, \bar{u}_{n}$ determine $Q$ in $F\left\{U_{1}, \cdots, U_{n}\right\}$, then $Q$ is a prime ideal of the type sought. Q.E.D.

While Ritt has not explicitly stated, in [6] at any rate, the strong form of Hilbert's Theorem, he does have, in a special case, a theorem much stronger than the weak form. In the case in question, $F$ is the field of functions meromorphic throughout a given open set $A$ of the complex plane. A point is then defined as the composite notion of an open set $B, B \subseteq A$, and a system of functions $u_{1}(x), \cdots, u_{n}(x), u_{i}(x)$ analytic in $B$. Hilbert's Theorem continues to hold. Applying the strong form of the theorem, one could prove this theorem using a minimum amount of analysis.

5. The chain theorem. Let $F$ be a differential field, $U_{1}, \cdots, U_{n}$ differential indeterminates. The ascending chain condition does not hold in $S=F\left\{U_{1}, \cdots, U_{n}\right\}$ for ideals in general, but it does hold for the perfect ideals. We give a new proof, first establishing the following (for ground-fields of characteristic 0 ).

THEOREM 2. (A) The ascending chain condition holds for prime ideals.

(B) Every perfect ideal is a finite intersection of primes.

We may remark that (A) can be proved very simply using the theory of transcendency, both differential and ordinary. We shall want Theorem 2 in a slightly more general situation, however, as in the following lemma.

LEMMA. Let $R$ be an integral domain (in the algebraic sense, and of arbitrary characteristic). If the conditions (A) and (B) hold in $R$, then they also hold in $R[U]$, where $U$ is a single algebraic indeterminate. If $R$ is a differential domain containing the rational numbers, then the like is true of $R$ and $R\{U\}$, where $U$ is a differential indeterminate.

Proof. Let $A=\cap P_{\alpha}$ be a perfect ideal in $S=R[U]$. The ideal $A^{\prime}=R \cap A$ contains some prime ideals, for example, $(0)$ : let $P^{\prime}$ be a maximal prime ideal in $R$ contained in $A^{\prime}$. Since the ascending chain condition holds for prime ideals in $R$, we may assume, inductively, that any perfect $S$-ideal whose contraction to $R$ contains a prime ideal properly containing $P^{\prime}$ is a finite intersection. Now $A^{\prime}$ is clearly perfect, so by induction $A^{\prime}=P_{1}^{\prime} \cap \cdots \cap P_{k}^{\prime}$. If $A^{\prime} \neq P^{\prime}$, then each $P_{i}^{\prime} \supset P^{\prime}$ properly. Let $A_{i}=\cap P_{\alpha}$ over those $P_{\alpha}$ containing $P_{i}^{\prime}$; then each $A_{i}$ is a finite intersection, whence $A=A_{1} \cap \cdots \cap A_{k}$ is also. Thus we may assume $A^{\prime}=P^{\prime}$. We now consider polynomials $G(U)=\sum c_{i} U^{i}$ $\in A$. Let $\bar{G}(U)=\sum \bar{c}_{i} U^{i}$, where the $\bar{c}_{i}$ are the residues of the $c_{i} \bmod P^{\prime}$. If all 
$\bar{G}(U)=0$, one sees easily that $A$ is prime, and is the extension of $P^{\prime}$ to $S$. We may suppose, then, that there are $G(U) \in A$ such that $\bar{G}(U) \neq 0$, and of these we choose one, $G(U)$, to be of minimal degree $d$. For each perfect $S$-ideal $A$ contracting to $P^{\prime}$ we have then defined an integer $d=d(A)$, and may assume inductively that any perfect $S$-ideal contracting to $P^{\prime}$ and having a degree less than $d$.is a finite intersection. Factor $\bar{G}(U)$ in $\Sigma[U]$, where $\Sigma$ = quotient-field of $R / P^{\prime}: \overline{A G}=\bar{G}_{1} \cdots \bar{G}_{s}$, where $\bar{G}_{i}(U)$ is irreducible in $\Sigma[U]$, and $\bar{A} \in R / P^{\prime}$ has been so selected that $\bar{G}_{i}$ has coefficients in $R / P^{\prime}$. Let $A_{i}=\cap P_{\alpha}$ over those $P_{\alpha}$ containing $G_{i}(U):$ if $s>1$, then either $A_{i}$ contracts in $R$ to something containing $P^{\prime}$ properly or the degree of $A_{i}$ is less than $d$; in either case $A_{i}$ is a finite intersection, and hence so is $A=A_{1} \cap \ldots \cap A_{8}$. Thus we may assume that $s=1$, or that $\bar{G}$ is irreducible. Let $I$ be the leading coefficient of $G: I \notin P^{\prime}$ by the minimal condition placed on $G$. Let $A_{1}=\cap P_{\alpha}$ over those $P_{\alpha}$ containing $I, A_{2}=\cap P_{\alpha}$ over those $P_{\alpha}$ not containing $I$. The ideal $A_{1}$ is a finite intersection, so we may suppose $A=A_{2}$, i.e., that $I \notin P_{\alpha}$ for every $\alpha$. Hence in particular $I^{\rho} H \in A$ implies $H \in A$. Under these circumstances, however, $A$ is even prime. In fact, let $H_{1}(U) H_{2}(U) \in A$. Reduce the degrees of $H_{1}, H_{2}: I^{\rho} H_{1} \equiv H_{1}^{\prime}(U)(G)$, $I^{\sigma} H_{2} \equiv H_{2}^{\prime}(U)(G)$, where $\operatorname{deg} H_{i}^{\prime}(U)<d, i=1,2$. We have $H_{1}^{\prime} H_{2}^{\prime} \in A$, and from $H_{i}^{\prime} \in A$ follows $H_{i} \in A$. Let $I^{\top} H_{1}^{\prime} H_{2}^{\prime} \equiv R(U)(G)$, where $\operatorname{deg} R(U)<d$. Then $\bar{I}^{r} \bar{H}_{1}^{\prime} \bar{H}_{2}^{\prime}$ is divisible by $\bar{G}$ in $\Sigma[U]$, and this implies $\bar{H}_{1}^{\prime}=0$ or $\bar{H}_{2}^{\prime}=0$, whence $H_{1}^{\prime} \in A$ or $H_{2}^{\prime} \in A$. Thus $A$ is prime, and the proof that a perfect ideal is a finite intersection is complete. The proof that the ascending chain condition holds for prime ideals in $S$ follows in quite the same way: in fact, one shows easily that if $S \cdot P^{\prime} \subset Q_{1} \subseteq Q_{2}$, where $Q_{1}, Q_{2}$ are prime ideals contracting to $P^{\prime}$ and $Q_{1} \supset S \cdot P^{\prime}$ properly, then $Q_{1}=Q_{2}$.

The proof of the second point is almost the same, and could easily be carried through without the introduction of any further general remark. The following corollary, which will be useful below, may, however, also be used here. Let $M$ be a set of prime ideals in a ring $R$ and define an $M$-perfect ideal as an intersection of $M$-prime ideals.

CoRollary. If conditions (A) and (B) hold for the $M$-prime and $M$-perfect ideals in an (algebraic) ring $R$, and $(0) \in M$, then they also hold for any set $N$ of prime ideals in $R[U]$ and their $N$-perfect ideals, provided that the contraction of an $N$-prime ideal is an $M$-prime ideal and that an $N$-perfect ideal which is prime is an $N$-prime ideal.

To apply the corollary to $R$ and $S=R\{U\}$, introduce the rings $S_{i}$ $=R\left[U, U_{1}, \cdots, U_{i}\right]$, and let the $M_{i}$-prime ideals be those prime ideals which are contractions of prime or perfect differential ideals in $R\{U\}$; in $R$, the $M$-prime ideals are the differential prime ideals. Then one sees that conditions (A) and (B) hold in $S_{i}$ for the $M_{i}$-ideals. Let now $A=\cap P_{\alpha}$ be a perfect ideal in $R\{U\}$ : as before we suppose that $A \cap R=P^{\prime}$ is prime, that the theorem holds for any perfect ideal whose contraction to $R$ contains $P^{\prime}$ 
properly, and that $A \neq R\{U\} \cdot P^{\prime}$. Let $G\left(U, U_{1}, \cdots\right) \in A$, with coefficients not all in $P^{\prime}$, and of minimal total degree in $U, U_{1}, \ldots$; we may, and shall, also suppose that none of the coefficients of $G$ is in $P^{\prime}$. Let $G=G\left(U, \cdots, U_{r}\right)$ effectively involve $U_{r}$, but not $U_{s}, s>r$, say $\operatorname{deg} G$ in $U_{r}$ is $d$. The leading coefficient of $G$, i.e., the coefficient of $U_{r}^{d}$, is not in $R\{U\} \cdot P^{\prime}$, hence also the leading coefficient of $S=\partial G / \partial U_{r}$ is not in $R\{U\} \cdot P^{\prime}$. Hence $S$ is not in $A$, being of too small degree. Thus $S$ is not in every $P_{\alpha}$, and separating off those $P_{\alpha}$ containing $S$, we may suppose $S \notin P_{\alpha}$, every $\alpha$ : in particular, then, $S^{\ominus} H \in A$ implies $H \in A$. We can now further suppose that $A \cap R\left[U, \cdots, U_{r}\right]$ $=P_{r}^{\prime}$ is prime. Under these circumstances, $A$ is prime. In fact, let $H_{1} H_{2} \in A$. Noting that $S$ is the coefficient of $U_{r+i}$ in $G^{(i)}$, we see that for some $\rho, \sigma$, $S^{\rho} H_{1} \equiv H_{1}^{\prime}(A), \quad S^{\sigma} H_{2} \equiv H_{2}^{\prime}(A)$, where $H_{1}^{\prime}, H_{2}^{\prime} \in R\left[U, \cdots, U_{r}\right]$. Since $H_{1}^{\prime} H_{2}^{\prime} \in A$, also $H_{1}^{\prime} H_{2}^{\prime} \in P_{r}^{\prime}, H_{1}^{\prime}$ or $H_{2}^{\prime}$ is in $P_{r}^{\prime}$, hence in $A$, and $H_{1}$ or $H_{2}$ is in $A$. This completes the proof for (A), and (B) follows similarly.

The chain theorem follows readily from (A) and (B), without reference to any special differential concept. For let $A_{1} \subseteq A_{2} \subseteq \cdots$ be a chain of perfect ideals, and let each $A_{i}$ be written as an irredundant intersection of a finite number of prime ideals. Let $P_{1} \subseteq P_{2} \subseteq \cdots$ be a chain of prime ideals, where $P_{i}$ occurs in $A_{i}$. Each such chain involves only a finite number, say $n$, of prime ideals. Then $n$ must be bounded. For if not, then there is some prime $P_{1}$ of $A_{1}$ which initiates, for any $n$, chains of length $\geqq n$. Take chains of length $n \geqq 1,2, \cdots$ and beginning with $P_{1}$. In all these we may have $P_{1}=P_{2}=\cdots$ $=P_{i}$, but there is some $i$ such that $P_{i} \subset P_{i+1}$ for some, and hence every, such chain (because $A_{i+1}$ has been written irredundantly). There are only a finite number of possibilities for $P_{i+1}$, so what has been said for $P_{1}$ also goes for some $P_{i+1}$ containing $P_{1}$ properly. In this way we get a proper ascending chain of prime ideals: impossible. So $n$ is bounded. Let there be chains of length $N$, but none of length greater than $N$. We make an induction on $N$. Each $P_{i+1}$ contains some $P_{i}$, and if $A_{i} \subset A_{i+1}$ properly, then either at least one $P_{i+1}$ contains some $P_{i}$ properly, or $A_{i+1}$ has less primes than $A_{i}:$ if $N=1$, then the first possibility is excluded, and the chain $A_{1} \subseteq A_{2} \subseteq \cdots$ is finite. Suppose now that $P_{1}=P_{2}=\cdots=P_{i} \subset P_{i+1} \subseteq \cdots$ is a chain of length $N>1$ : call the pair $\left(P_{i}, i\right)$ an initiator. If $\left(P_{i}, i\right),\left(P_{j}, j\right)$ are initiators and $i \neq j$, say $j>i$, then also $P_{i} \neq P_{j}$, for otherwise $P_{j}\left(=P_{i}\right)$ would be a prime ideal of $A_{i+1}$, and that is not so. Each $P_{i}$ of an initiator is a prime ideal of $A_{1}$, so there are at most a finite number of initiators. Let $i$ be maximal over the indices of the initiators. Then the theorem follows by induction on $A_{i+1} \subseteq A_{i+2} \subseteq \cdots \cdot$

6. The case of characteristic $p \neq 0$. To extend the results of $\S 2$ one must first decide what one shall mean by the element $u$ being algebraic over a differential field. Let $\pi$ be the prime field of ch. $p \neq 0, x$ an indeterminate in the algebraic sense. Convert $F=\pi(x)$ into a differential field by setting each derivative equal to zero. In the polynomial ring $S=F\{U\}$, consider the ideal $P=S \cdot\left(U^{p}-x\right)$, generated in the algebraic sense. One sees that $P$ is a prime 
differential ideal, that the residue $u$ of $U$ in $S / P$ is algebraic in the previous sense, but that $u^{\prime}$ is not algebraic in that sense. Here $S / P$ has infinite degree of transcendence (in the algebraic sense) over $F$. One ought at least to require of an algebraic quantity $u$ that $F\langle u\rangle$ be of finite degree of transcendence over $F$, but this is not sufficient, as axiom II would fail.

ExAmpLE. Let $\pi$ be the prime field of ch. $p \neq 0$. Let $x, x_{1}, \cdots$ be a sequence of indeterminates in the algebraic sense, and convert $F=\pi\left(x, x_{1}, \cdots\right)$ into a differential field by setting every derivative equal to zero. In the ring $S=F\{V\}$, consider the ideal $P=S \cdot\left(V^{p} x_{1}-V_{1}^{p} x, V_{2}^{p} x_{3}-V_{3}^{p} x_{2}, \cdots\right)$ generated in the algebraic sense $\left(V_{1}=V^{\prime}, V_{2}=V^{\prime \prime}, \cdots\right)$. One verifies immediately that $P$ is a prime differential ideal: in fact, if $t, t_{2}, t_{4}, \cdots$ is a sequence of indeterminates (in the algebraic sense), then $\left(t, t\left(x_{1} / x\right)^{1 / p}, t_{2}, t_{2}\left(x_{3} / x_{2}\right)^{1 / p}, \cdots\right)$ is a "general point" of $P$. If $v, v_{1}, \cdots$ are the residues of $V, V_{1}, \cdots$ in $S / P$, one sees that $F\langle v\rangle$ is not of finite degree of transcendence over $F$. In $T$ $=F\langle v\rangle\{U\}$, let $Q=T \cdot\left(x U^{p}-v^{p}, x_{2} U_{2}^{p}-v_{2}^{p}, \cdots\right)$, generated in the algebraic sense. Here also $Q$ is a differential prime ideal in $T$, and if $u$ is the residue of $U$ in $T / Q$, then $F\langle v, u\rangle$ is of infinite degree of transcendence over $F\langle v\rangle$. On the other hand, each $v_{i}$ is algebraic over $F\langle u\rangle$.

We therefore propose the following definition.

Definition. The element $u$ is said to be algebraic over $F$ if $F\langle u\rangle$ is a finite extension of $F$.

The results of $\$ 2$ then continue to hold. Axiom I is trivially verified; III follows from the fact that if $K, L, M$ are fields, $K \subseteq L \subseteq M$, and $M / K$ is finite, then so is $L / K$. As for II, adjoining $u_{1}, \cdots, u_{n-1}$ to $F$, and calling the other two elements $u, v$, we have to see that if $v$ is algebraic over $F\langle u\rangle$ but not over $F$, then $u$ is algebraic over $F\langle v\rangle$. Let, then, $F\langle u, v\rangle=F\langle u\rangle\left(v, v_{1}, \cdots, v_{r}\right)$. We have $v_{r+1}=P\left(u, u_{1}, \cdots, u_{t}, v, \cdots, v_{r}\right) / Q\left(u, \cdots, u_{t}, v, \cdots, v_{r}\right), P, Q$ polynomials. Let us use this relation to compute $v_{r+2}, v_{r+3}, \cdots$, the denominators being always powers of $Q$. In computing $v_{r+2}, v_{r+1}$ and $u_{t+1}$ may arise in the numerator: eliminating $v_{r+1}$ by the above relation, we have $v_{r+2}=P_{1}\left(u, \cdots, u_{t}, u_{t+1}, v, \cdots, v_{r}\right) / Q^{p}$, and the degree of $P_{1}$ in $u_{t+1}$ is at most 1 . Here $P_{1}$ may not actually involve $u_{t+1}$, but at any rate one sees that the order of the highest order derivative of $u$ appearing in the numerator increases by 0 or 1 (or possibly decreases); and the like is true in passing successively to $v_{r+3}, v_{r+4}, \cdots$. Now the order of this highest derivative can not be bounded, as otherwise $F\langle v\rangle$ would be finite over $F$. And since this order increases by either 0 or 1 , one sees that it takes on all the values $t, t+1$, $t+2, \cdots$. The increase from $u_{s}$ to $u_{s+1}$ gives a linear relation in $u_{s+1}$, and so we have that $F\langle v, u\rangle$ is a finite extension of $F\langle v\rangle$, i.e., $u$ is algebraic over $F\langle v\rangle$.

To extend the results of $\S 3$ one might think, keeping in mind the abstract algebraic results, that the first thing is to define separable quantity. It turns out, however, that the theorem of the primitive element has nothing to do with questions of separability, at least subject to the definition of algebraic 
quantity given above. In fact, if $u$ and $v$ are each algebraic over $F$, then $F\langle u, v\rangle / F$ as well as $F\langle\Lambda\rangle\langle u, v\rangle / F\langle\Lambda\rangle$ are finite. Now any subfield of a finite extension is also finite, and from any system of generators, a finite subsystem of generators can be selected. Hence $u+\Lambda v$ is algebraic over $F\langle\Lambda\rangle$ and for some $r,(u+\Lambda v)^{(r)} \in F\langle\Lambda\rangle\left(u+\Lambda v,(u+\Lambda v)^{\prime}, \cdots,(u+\Lambda v)^{(r-1)}\right)$. As before, taking a partial with respect to $\Lambda^{(r)}$, one obtains $F\langle\Lambda\rangle\langle u+\Lambda v\rangle=F\langle\Lambda\rangle\langle u, v\rangle$.

The results of $\$ 3$ would now carry over provided we have the theorem on satisfying polynomial inequalities. Here again the theorem depends on the nature of the base field, but it is not sufficient to have a nonconstant element.

ExAmple. Let $\pi$ be the prime field of ch. 2, $\pi(\xi)$ a differential field with $\xi^{\prime}=1$. Then $\Lambda^{\prime \prime}$ is a nonzero polynomial which vanishes for every $\lambda \in \pi(\xi)$.

If $F$ is a differential field, then the constants in $F$ form a field $F_{0}$, the constant field. If an element $\xi$ in $F$ is separable over $F_{\theta}$ (in the algebraic sense), then one sees immediately that $\xi \in F_{0}$. In the case of ch. 0 , if $\xi$ is nonconstant, then this shows that $\xi$ is transcendental over $F_{0}$, in particular $F / F_{0}$ has no finite linear basis. This is the main condition that the theorem on satisfying polynomial inequalities hold.

THEOREM 3. The theorem on satisfying polynomial inequalities over $F$ holds if and only if $F$ has no finite linear basis over its constant field $F_{0}$.

Proof. By a lemma of Ritt [6, p. 34], the proof of which is a simple induction and in no way depends on the characteristic, elements $\eta_{1}, \cdots, \eta_{s} \in F$ are linearly dependent over $F_{0}$ if and only if

$$
\left|\begin{array}{ccc}
\eta_{1} & \cdots & \eta_{s} \\
\eta_{1}^{\prime} & \cdots & \eta_{s}^{\prime} \\
& \vdots & \\
\eta_{1}^{(8-1)} & \cdots & \eta_{s}^{(8-1)}
\end{array}\right|=0 .
$$

Let $F / F_{0}$ be finite, and let $s>\left[F: F_{0}\right]$. Form a polynomial $P(\Lambda)$ by replacing in the above determinant $\Lambda^{(i)}$ for $\eta_{i}$. Then $P(\Lambda) \neq 0$, but $P(\lambda)=0$ for every $\lambda \in F$. Conversely, let $G\left(U, \cdots, U_{s}\right) \neq 0$ be a given polynomial, and let $\xi_{0}, \cdots, \xi_{s} \in F$ be linearly independent over $F_{0}$ : we propose to show that $G \neq 0$ is satisfied for some $U=c_{0} \xi_{0}+\cdots+c_{s} \xi_{s}$, the $c_{i} \in F_{0}$. In doing so, we may assume inductively that the theorem holds for any polynomial (involving only $U, \cdots, U_{s}$ ) of total degree (in $U, \cdots, U_{s}$ ) less than the total degree of $G$ : the theorem being trivial for total degree zero, we shall suppose $G$ to have positive degree. Suppose, then, that $G$ were also a polynomial in $U^{p}, \cdots, U_{s}^{p}$. Let $w_{1}, w_{2}, \cdots$ be a (possibly infinite) linear basis of $F / F^{p}$. Then $G=G_{1} w_{1}$ $+G_{2} w_{2}+\cdots$, where $G_{i} \in F^{p}\left[U^{p}, \cdots, U_{s}^{p}\right]$. Let $G_{i}(U) \neq 0$ and $U=c_{0} \xi_{0}$ $+\cdots+c_{s} \xi_{s}$ such that $G_{i}^{1 / p}\left(c_{0} \xi_{0}+\cdots+c_{s} \xi_{s}\right) \neq 0$ : such an element exists by induction, and also does not annihilate $G$. We may suppose, then, that $G$ is 
not a polynomial in $U^{p}, \cdots, U_{s}^{p}$, which we can express by saying that some $\partial G / \partial U_{i} \neq 0$. The proof is now parallel to Ritt's, where he has written $1, \xi, \cdots, \xi^{s}$ instead of $\xi_{0}, \cdots, \xi_{s}$. In fact, suppose $G\left(c_{0} \xi_{0}+\cdots+c_{s} \xi_{s}\right)=0$ for all $\left(c_{0}, c_{1}, \cdots, c_{s}\right), c_{i} \in F_{0}$. Let $\pi$ be the prime field of $F$, and $\xi \in F, \xi \notin F_{0}$. Then $\xi$ is not algebraic over $\pi$ (in the algebraic sense): in fact, suppose for a moment it were, and let $f(X)=0$ be an equation of least degree satisfied by $\xi$ over $\pi$. Then $f(X)$ is separable, since $\pi$ is perfect, so $f^{\prime}(\xi) \cdot \xi^{\prime}=0$ yields $\xi^{\prime}=0$, a contradiction. Hence in particular $F_{0}$ contains infinitely many elements, all the elements of $\pi\left(\xi^{p}\right)$ for example. Hence taking the partials of $G\left(c_{0} \xi_{0}+\cdots+c_{s} \xi_{s}\right)=0$ with respect to the $c_{i}$ yields true relations. Thus we get:

$$
\begin{gathered}
\frac{\partial G}{\partial U} \xi_{0}+\frac{\partial G}{\partial U_{1}} \xi_{0}^{\prime}+\cdots+\frac{\partial G}{\partial U_{s}} \xi_{0}^{(s)}=0, \\
\frac{\partial G}{\partial U} \xi_{1}+\frac{\partial G}{\partial U_{1}} \xi_{1}^{\prime}+\cdots+\frac{\partial G}{\partial U_{s}} \xi_{1}^{(s)}=0, \\
\vdots \\
\frac{\partial G}{\partial U} \xi_{s}+\frac{\partial G}{\partial U_{1}} \xi_{s}^{\prime}+\cdots+\frac{\partial G}{\partial U_{s}} \xi_{s}^{(s)}=0,
\end{gathered}
$$

whence, since $\partial G / \partial U_{i} \neq 0$ for an appropriate choice of the $c_{i}$, we get that the determinant of these equations equals zero, and hence that $\xi_{0}, \cdots, \xi_{s}$ are linearly dependent over $F_{0}$, by the lemma of Ritt already referred to above: this is a contradiction.

Hence, the theorem of $\$ 3$ on the primitive element also holds more generally, with a condition on the base field $F$, but without restriction on the characteristic.

As for the chain theorem, we must first settle on the definition of prime ideal: the old definition is certainly not sufficient.

EXAMPLE. Let $\pi=$ the prime field of ch. $p \neq 0, x, x_{1}, \cdots$ a sequence of (algebraic) indeterminates, and $F=\pi\left(x, x_{1}, \cdots\right)$ a differential field in which every derivative is zero. In $S=F\{U\}$, let $P_{i}=S \cdot\left(U^{p}-x, \cdots, U_{i}^{p}-x_{i}\right)$, generated in the algebraic sense: one sees that also $P_{i}$ is differential and prime. On the other hand $P \subset P_{1} \subset \ldots$ is an infinite ascending chain of prime ideals.

In this example, $S / P$ is of infinite degree of transcendence over $F$. Even requiring $S / P$ to be finite is not sufficient, however. The chain theorem for prime ideals would, indeed, obtain, but the finite intersection property fails.

ExAmple. Let $S$ be as above; let $P_{i}=S \cdot\left(U^{p}-x, \cdots, U_{i-1}^{p}-x_{i-1}, U_{i}\right.$, $\left.U_{i+1}, \cdots\right)(i=1,2, \cdots ; \omega)$ in the algebraic sense. One verifies immediately that $P_{i}$ is a differential prime ideal. On the other hand, $A=\cap P_{i}$ is not a finite intersection. In fact, $A=S \cdot\left[U\left(U^{p}-x\right), U_{1}\left(U_{1}^{p}-x_{1}\right), \cdots\right]$, generated 
in the differential sense, i.e., these generators and their various derivatives generate $A$ in the algebraic sense. The minimal prime ideals of $A$ are just the $P_{i}$, so $A$ cannot be a finite intersection.

Thus it becomes fairly clear that at least in $S=F\{U\}$ it should be required of an allowable proper prime ideal that $F\langle u\rangle / F$, where $u$ is the residue of $U \bmod P$, satisfy some separability condition, in addition to the requirement that it be finite. We take the following definition, which is the current one in the algebraic theory (see $[1$, p. 68]), and which appears to be suitable for the differential theory as well.

Definition. Let $K \subseteq L, K, L$, be fields (of ch. $\mathrm{p} \neq 0$ ). $L / K$ is said to be separable if elements in $L$ linearly independent over $K$ are still such over $K^{1 / p}$.

We define allowable prime ideal accordingly.

Definition. A differential prime ideal $P$ in $S=F\left\{U_{1}, \cdots, U_{n}\right\}$ will be said to be allowable if $F\left\langle u_{1}, \cdots, u_{n}\right\rangle / F$ is separable, where $F\left\{u_{1}, \cdots, u_{n}\right\}$ $=S / P$.

In $S=F\left\{U_{1}, \cdots, U_{n}\right\}$, we define an allowable perfect ideal as the intersection of allowable prime ideals, but it is not immediately clear that an allowable perfect ideal which is prime is an allowable prime ideal. We need an idealtheoretic or ring-theoretic criterion for an allowable ideal. Let $z_{1}, z_{2}, \cdots \in F$ be a $p$-basis for $F / F^{p}$, i.e., every element of $F$ can be written uniquely as a polynomial in the $z_{i}$, with no exponent exceeding $p-1$, and with coefficients in $F^{p}$. One easily defines differentiations $\partial / \partial z_{i}$ over $F^{p}$ such that $\partial z_{i} / \partial z_{i}=1$, $\partial z_{j} / \partial z_{i}=0$ if $j \neq i$, and $\partial U_{j} / \partial z_{i}=0$.

Theorem 4. Let $P$ be a prime ideal in $S=F\left\{U_{1}, \cdots, U_{n}\right\}$. Then $P$ is allowable if and only if $P \cap F\left[U_{1}^{p}, U_{1}^{\prime p}, \ldots, U_{n}^{p}, U_{n}^{\prime p}, \cdots\right]$ is closed under all the differentiations $\partial / \partial z_{i}$.

Proof. Let $u_{1}, \cdots, u_{n}$ be the residues of $U_{1}, \cdots, U_{n} . F\left\langle u_{1}, \cdots, u_{n}\right\rangle / F$ is separable if and only if $F^{p}\left(u_{1}^{p}, u_{1}^{\prime p}, \cdots, u_{n}^{p}, u_{n}^{\prime p}, \cdots\right) / F^{p}$ is separable, and this will be the case if and only if $G_{1} w_{1}+G_{2} w_{2}+\cdots=0$ implies that each $G_{i}=0$, where $G_{i} \in F^{p}\left(u_{1}^{p}, \ldots\right)$ and $w_{1}, w_{2}, \cdots$ is a linear basis of $F / F^{p}$. We may suppose that $G_{i} \in F^{p}\left[u_{1}^{p}, \cdots\right]$, and the $w_{i}$ to be the power products of the $z_{i}$ with exponents not exceeding $p-1$. The differentiations $\partial / \partial z_{i}$ are introduced merely as a convenient device for deducing $G_{i}=0$ from $G_{1} w_{1}+G_{2} w_{2}+\cdots=0$. ideal.

COROllaRy. An allowable perfect ideal which is prime is an allowable prime

REMARK. The above ought, no doubt, to be the basis of defining an allowable ideal in general. If we do take this definition in general, then we recover Hilbert's Nullstellensatz (weak form) as a theorem. In fact, the leading statements in the above proof are still true if [ ] and \{\} are taken in the (present) narrower sense, but these do require some further substantiation. Let $A, P$ 
be as before, except that now they are allowable. Let $A_{0}$ be exactly as before, i.e., generated in the (previous) wider sense by $P$ and $u$; and similarly for $B_{0}$. As before we have $A_{0} \cdot B_{0} \subseteq P$. Let $A_{0}^{\prime}$ be generated in the wider sense by $A_{0}$ and the partials with respect to the $z_{i}$ of the elements in $A_{0} \cap F\left[U_{1}^{p}, U_{2}^{p}, \ldots\right]$; and similarly for $B_{0}^{\prime}$. Let $C \in A_{0} \cap F\left[U_{1}^{p}, U_{2}^{p}, \cdots\right], D \in B_{0}$. Then $\partial\left(C D^{p}\right) / \partial z_{i}$ $=D^{p} \cdot \partial C / \partial z_{i} \in P$, whence $\left(D \cdot \partial C / \partial z_{i}\right)^{p} \in P$ and $D \cdot \partial C / \partial z_{i} \in P$. Using the calculation proving $A_{0} \cdot B_{0} \subseteq P$, we see that $A_{0}^{\prime} \cdot B_{0} \subseteq P$; and repeating the argument, that $A_{0}^{\prime} \cdot B_{0}^{\prime} \subseteq P$. Let $A_{1}$ be the ideal generated in the wider sense by the $G$ such that $G^{\rho} \in A_{0}^{\prime}$ for some $\rho$; and similarly for $B_{1}$. As before we get $A_{1} \cdot B_{1} \subseteq P$. Defining $A_{i}, B_{i}, A_{i}^{\prime}, B_{i}^{\prime}$ recursively, and repeating the above argument an infinite number of times, we get $\{P, u\} \cdot\{P, v\} \subseteq P$.

We are now in position to prove the chain theorem.

THEOREM 5. In the case of ch. $p \neq 0$, conditions $(\mathrm{A})$ and (B) continue to hold for the allowable prime and perfect ideals.

Proof. Let $A=\cap P_{\alpha}$ be an allowable perfect ideal in $S=F\left\{U_{1}, \cdots, U_{n}\right\}$; we may suppose $A \neq(0)$. Let $G \in A, G \neq 0, G$ of minimal degree in $U_{1}, \cdots, U_{n}$ and its derivatives: this degree may, trivially, be supposed positive, and further, we may assume inductively that every allowable perfect ideal containing an element of smaller degree is a finite intersection. Now $G$ $\notin F\left[U_{1}^{p}, U_{1}^{\prime p}, \cdots, U_{n}^{p}, U_{n}^{\prime p}, \cdots\right]$. For if it were, then $G=G_{1} w_{1}+G_{2} w_{2}+\cdots$, where $w_{1}, w_{2}, \cdots$ is a linear basis of $F / F^{p}$, and $G_{i} \in F^{p}\left[U_{1}^{p}, \cdots\right]$. Since $A$ is allowable, all the $G_{i} \in A$, hence $G_{i}^{1 / p} \in A$ and not all $G_{i}=0$; this contradicts the minimum condition on $G$. So at least one of the variables in $G$ occurs with exponent not divisible by $p$, say $U_{n r}$, the $r$ th but no higher derivative of $U_{n}$, occurs with such exponent $t: U_{n s}, s>r$, may occur, but with exponent divisible by $p$. It is convenient, and we may assume, that no such terms $U_{n s}, s>r$, occur; namely, adjoining (for a moment) $U_{n s}^{p}, s>r$, to the groundfield, we see that the coefficient of $U_{n, r+1}$ in $G^{\prime}$ is $\partial G / \partial U_{n r}$, in particular then $G^{\prime} \neq 0$, and $U_{n, r+1}$ occurs with exponent not divisible by $p$; $\operatorname{deg} G^{\prime}=\operatorname{deg} G$, and we replace $G$ by $G^{\prime}$. Replacing $G$ by a still higher derivative if necessary, we may assume that $\operatorname{deg} G=0$ in $U_{n s}, s>r$. The coefficient of $U_{n r}^{t}$, being of too small degree, is not in $A$; it may be in some of the $P_{\alpha}$, but separating these off, we may suppose that it is not in any $P_{\alpha}$. As in the ch. 0 case, we may suppose the theorem to hold for $n-1$ variables, and even that the contraction of $A$ to $F\left\{U_{1}, \cdots, U_{n-1}\right\}\left[U_{n}, \cdots, U_{n r}\right]$ is prime; here we make use of the corollary to $\S 5$ just as in the proof of the second point of the lemma. Under these circumstances, $A$ is prime, and the proof for (A) is complete; (B) follows similarly.

For an allied discussion of the above, see Kolchin [3].

7. Separable extensions. According to our definition of an algebraic quantity, it is quite possible for an element $u$ to be nonalgebraic over $F$ and yet each $u_{i}$ be algebraic (in the algebraic sense) over $F$. 
ExAmple. Let $F=\pi\left(x, x_{1}, \cdots\right), \pi=$ prime field of ch. $p \neq 0, x, x_{1}, \cdots$ a sequence of (algebraic) indeterminates, and let $\alpha^{\prime}=0$ for every $\alpha \in F$. In $S=F\{U\}$, let $P=S \cdot\left(U^{p}-x, U_{1}^{p}-x_{1}, \cdots\right)$. Then the residue of $U \bmod P$ has the desired properties.

This pathological feature does not appear in the case of separable extensions. For explicitness we write out the definition of separable quantity.

Definition. The algebraic differential quantity $u$ is said to be separable over $F$ if $F\langle u\rangle / F$ is separable.

THEOREM 6. If $u$ is transcendental over $F$ and $F\langle u\rangle / F$ is separable, then $u, u_{1}, u_{2}, \cdots$ are algebraically independent (in the algebraic sense) over $F$.

Proof. Suppose there were a nontrivial relation $G\left(u_{1}, \cdots, u_{r}\right)=0$; then let $G\left(U, \cdots, U_{r}\right)$ be of minimal total degree. If $G \notin F\left[U^{p}, U_{1}^{p}, \cdots\right]$, then one sees that we may assume $G$ to have a term with $U_{r}$ occurring with exponent not divisible by $p$ (by replacing $G$ by a derivative, as in the proof of Theorem 5). In that event, however, $F\langle u\rangle=F\left(u, \cdots, u_{r}, u_{r+1}\right)$. Suppose then that $G \in F\left[U^{p}, U_{1}^{p}, \cdots\right]$. Let $w_{1}, w_{2}, \cdots$ be a basis of $F / F^{p}$. Then $G(U)=G_{1}(U) w_{1}$ $+G_{2}(U) w_{2}+\cdots=0$, with $G_{i} \in F^{p}\left[U^{p}, U_{1}^{p}, \cdots\right]$, not all $G_{i}(U)=0$, whence also not all $G_{i}(u)=0$. On the other hand, $F^{p}\left(u^{p}, u_{1}^{p}, \cdots\right) / F^{p}$ is also clearly separable, so $w_{1}, w_{2}, \cdots$ are also linearly independent over $F^{p}\left(u^{p}, u_{1}^{p}, \cdots\right)$, a contradiction.

In precisely the same way one can establish the following.

Corollary. If d.d.t. $F\left\langle u_{1}, \cdots, u_{n}\right\rangle / F=n$ and $F\left\langle u_{1}, \cdots, u_{n}\right\rangle / F$ is separable, then the $u_{i j}$ are algebraically independent over $F$.

The situation for a separable algebraic quantity parallels the case of characteristic 0 .

THEOREM 7. Let $u$ be separable over $F$, and let d.t. $F\langle u\rangle / F=r$. Then $u, u_{1}, \cdots, u_{r-1}$ are algebraically independent (in the algebraic sense) over $F, u_{r}$ is separable over $F\left(u, \cdots, u_{r-1}\right)$, and $F\langle u\rangle=F\left(u, u_{1}, \cdots, u_{r}\right)$.

Proof. Let $u, u_{1}, \cdots, u_{s-1}$ be algebraically independent, but $u, \cdots, u_{s}$ algebraically dependent over $F$. Let $G\left(U, \cdots, U_{s}\right), G \neq 0$, be a polynomial of least degree satisfied by $u, \cdots, u_{s}$, the coefficients of $G$ being in $F$. As in the proof of the previous theorem, we may assume $U_{s}$ occurs with an exponent not divisible by $p$; i.e., we conclude that $F\langle u\rangle=F\left(u, \cdots, u_{s}\right)$, whence $s=r$, and the proof is complete.

Before proving the theorem of MacLane, we would like to consider two examples which are somewhat related to the point in question.

ExAmple. In the polynomial ring $S=F\{U, V\}$, the ideal $P$ $=\left[U^{p}-V_{1}, V^{p}-U_{1}\right]$ is prime. Let $S / P=F\{u, v\}$. Then $F\langle u, v\rangle=F(u, v)$. This field is separable over $F$, but $v$ is not separable over $F\langle u\rangle$, nor is $u$ separable over $F\langle v\rangle$. 
EXAMPLE. The sum of separable quantities is not necessarily separable. Let $\pi$ be the prime field of ch. $p>2, a, b$ algebraic indeterminates, and let $F=\pi(a, b)$, with $\alpha^{\prime}=0$ for every $\alpha \in F, S=F\{U, V\}$, a polynomial ring. One verifies that $P=\left[\left(U+V^{2}\right)^{p}+a\left(U+2 V^{2}\right)^{p}-b, U-U_{1}, V-V_{1}\right]$ is prime. In the residue class ring $F\{u, v\}(=F[u, v]), u$ is transcendental (in the algebraic sense), over $F$, so is $v ; u$ is separable, satisfying $U=U_{1}$, and $v$ also is separable. Moreover $\left(v^{2}\right)^{\prime}=2 v^{2}$, whence $v^{2}$ is separable; but $w=u+v^{2}$ is not separable. In fact $\left(u+v^{2}\right)^{p}+a\left(u+v^{2}\right)^{p}-b=-a v^{2 p}$, so $w$ is not algebraic (in the algebraic sense) over $F$. Now $w$ satisfies the irreducible polynomial $W^{p}+a W_{1}^{p}-b$; so $w$ is not separable over $F$.

TheOREM OF S. MAcLANE. Let $F\left\langle u_{1}, \cdots, u_{n}\right\rangle / F$ be separable and of degree of differential transcendency $t$. Then for some relettering of the $u_{i}, F\left\langle u_{1}, \cdots, u_{n}\right\rangle$ is separable over $F\left\langle u_{1}, \cdots, u_{t}\right\rangle$.

Proof. If $t=n$, there is nothing to prove. Let $t<n$, so that there exist nontrivial relations of the $u_{i}$ over $F$. Let $G\left(U_{1}, \cdots, U_{n}\right) \neq 0$ be such that $G\left(u_{1}, \cdots, u_{n}\right)=0$, and let $G$ be of minimum degree in the $U_{i j}$. Because $F\left\langle u_{1}, \cdots, u_{n}\right\rangle / F$ is separable and $G$ is of minimal degree, we know that at least one $U_{i j}$ occurs with exponent not divisible by $p$; say this is $U_{n r}$. Now drop the minimal condition of the degree of $G$, but assume that $r$ is minimal; i.e., $u_{n s}$ is separable over $F\left\langle u_{1}, \cdots, u_{n-1}\right\rangle\left(u_{n}, \cdots, u_{n, 8-1}\right)$ for $s=r$ but this is not the case for $s<r$. If now there are no nontrivial relations between the $u_{i j}, i<n$ or $i=n, j<r$, then we are through, since obviously $t=n-1$ and $u_{1}, \cdots, u_{n-1}$ is the required separating transcendency basis. Suppose, then, that there are such nontrivial relations, or that $t<n-1$. Let $G\left(u_{1}, \cdots, u_{n}\right)$ $=0$ be such a relation, where $G\left(U_{1}, \cdots, U_{n}\right)$ is of minimal degree. (Incidentally, a straightforward induction does not seem to work.) Again we know that at least one $U_{i j}, i<n$, occurs with exponent not divisible by $p$; say this is $U_{n-1, r_{1}}$. Now drop the minimal condition on the degree of $G$ but assume that $r_{1}$ is minimal; i.e., that $u_{n-1, s}$ is separable over $F\left\langle u_{1}, \cdots, u_{n-2}\right\rangle\left(u_{n}, \cdots, u_{n, r-1}\right)$ $\left(u_{n-1}, \cdots, u_{n-1,8-1}\right)$ for $s=r_{1}$, but this is not the case for $s<r_{1}$. If now there are no nontrivial relations between the $u_{i j}, i<n-1$, or $i=n-1, j<r_{1}$, or $i=n$, $j<r$, then we are through, since obviously $t=n-2$ and $u_{1}, \cdots, u_{n-2}$ is the required separating transcendency basis. If, however, $t<n-2$, then the argument is to be repeated, and the proof will be complete after $n-t$ applications of the argument.

8. The strong Nullstellensatz for arbitrary characteristic. The strong Nullstellensatz does not hold for arbitrary prime ideals: in fact, above $(\$ 7$, first example) we gave an example of a 1-dimensional prime ideal $P$ in $R$ $=F\{U\}$ for which $R / P$ is a field. The theorem holds, however, for separable prime ideals; and in fact, the previous proof also holds here, since what is needed is a separating transcendency basis, and this we have. The following proof, however, may also be of interest. 
Proof. Let $R / P=F\left\{u_{1}, \cdots, u_{n}\right\}$, and let $u_{1}, \cdots, u_{r}$ be algebraically independent over $F$. Since $F\left\langle u_{1}, \cdots, u_{r}\right\rangle / F$ is separable and of degree of differential transcendency $r$, one sees that the $u_{i j}, i=1, \cdots, r$, satisfy no nontrivial polynomial relations over $F$. One can now pick from the $u_{i j}, i>r$, a transcendency basis of $F\left\langle u_{1}, \cdots, u_{n}\right\rangle / F\left\langle u_{1}, \cdots, u_{r}\right\rangle$, say $v_{1}, v_{2}, \cdots, v_{s}$, and a linear basis $w_{1}, \cdots, w_{t}$ of $F\left\langle u_{1}, \cdots, u_{n}\right\rangle / F\left\langle u_{1}, \cdots, u_{r}\right\rangle\left(v_{1}, \cdots, v_{s}\right)$ from the power products of the $u_{i j}, i>r$. Then there exists a $d \in F\left\{u_{1}, \cdots, u_{r}\right\}\left[v_{1}, \cdots, v_{s}\right]$ such that for any $G \in F\left\{u_{1}, \cdots, u_{n}\right\}$ we can write $d^{\rho} G$ as a linear combination of the w's with coefficients in $F\left\{u_{1}, \cdots, u_{r}\right\}\left[v_{1}, \cdots, v_{s}\right]$. The element $d$ involves a number of derivatives of $u_{r}$, say up to $u_{r k}$. Take $g \geqq k$ and also so large that $u_{r}^{(o+1)}, u_{r}^{(0+2)}, \ldots$ be algebraically independent over $S=F\left\{u_{1}, \cdots, u_{r-1}\right\}\left[v_{1}, \cdots, v_{s}, w_{1}, \cdots, w_{t}\right.$, $\left.u_{r}, \cdots, u_{r g}\right]$; this will be so if $w_{1}, \cdots, w_{t}$ are algebraic (in the algebraic sense) over $F\left\{u_{1}, \cdots, u_{r-1}\right\}\left[v_{1}, \cdots, v_{s}, u_{r}, \cdots, u_{r g}\right]$. Let $S^{*}=\left\{\alpha / d^{\rho} \mid \alpha \in S\right.$, $\rho=1,2, \cdots\}$. Then $S^{*}\left\{u_{r}^{(\theta+1)}\right\}$ is a differential subring of $F\left\langle u_{1}, \cdots, u_{n}\right\rangle$. Let $Q^{*}$ be the ideal generated in the algebraic sense in $S^{*}\left\{u_{r}^{(\theta+1)}\right\}$ by $\left(u_{r}^{(\theta+1)}, u_{r}^{(\theta+2)}, \ldots\right)$; then $Q^{*}$ is clearly a proper prime ideal, and moreover is differential. Let $q=Q^{*} \cap F\left\{u_{1}, \cdots, u_{n}\right\}$. Then $q$ is a proper prime ideal in $F\left\{u_{1}, \cdots, u_{n}\right\}$, and it determines a proper prime ideal $Q \supset P$ in $F\left\{U_{1}, \cdots, U_{n}\right\}$; if $g$ is taken sufficiently large (so that $d^{p} \alpha(u) \in S$ ), then clearly also $\alpha(U) \notin Q\left(\alpha(U) \notin P\right.$, given). The residue class ring of $Q^{*}$ is just (isomorphic, in the abstract sense, to) $S^{*}$, and $F\left\{u_{1}, \cdots, u_{n}\right\} / q$ can be regarded as a subring of $S^{*}$; the quotient field of $S^{*}$ is a subfield of the separable field $F\left\langle u_{1}, \cdots, u_{n}\right\rangle$, whence it is separable and $Q$ is allowable. This completes the proof.

\section{REFERENCES}

1. C. Chevalley, Some properties of ideals in rings of power series, Trans. Amer. Math. Soc. vol. 55 (1944) pp. 68-84.

2. E. R. Kolchin, Extensions of differential fields. I, Ann. of Math. vol. 43 (1942) pp. 724729.

3. - On the basis theorem for differential fields, Trans. Amer. Math. Soc. vol. 52 (1942) pp. 115-127.

4. S. MacLane, Modular fields. I. Separating transcendence bases, Duke Math. J. vol. 5 (1939) pp. 372-393.

5. H. W. Raudenbush, Differential fields and ideals of differential forms, Ann. of Math. vol. 34 (1933) pp. 509-517.

6. J. F. Ritt, Differential algebra, Amer. Math. Soc. Colloquium Publications, vol. 33, New York, 1950.

7. B. L. van der Waerden, Moderne Algebra, vol. 1, Berlin, 1931.

8. A. Weil, Foundations of algebraic geometry, Amer. Math. Soc. Colloquium Publications, vol. 29, New York, 1946.

University of California, Berkeley, Calif. 